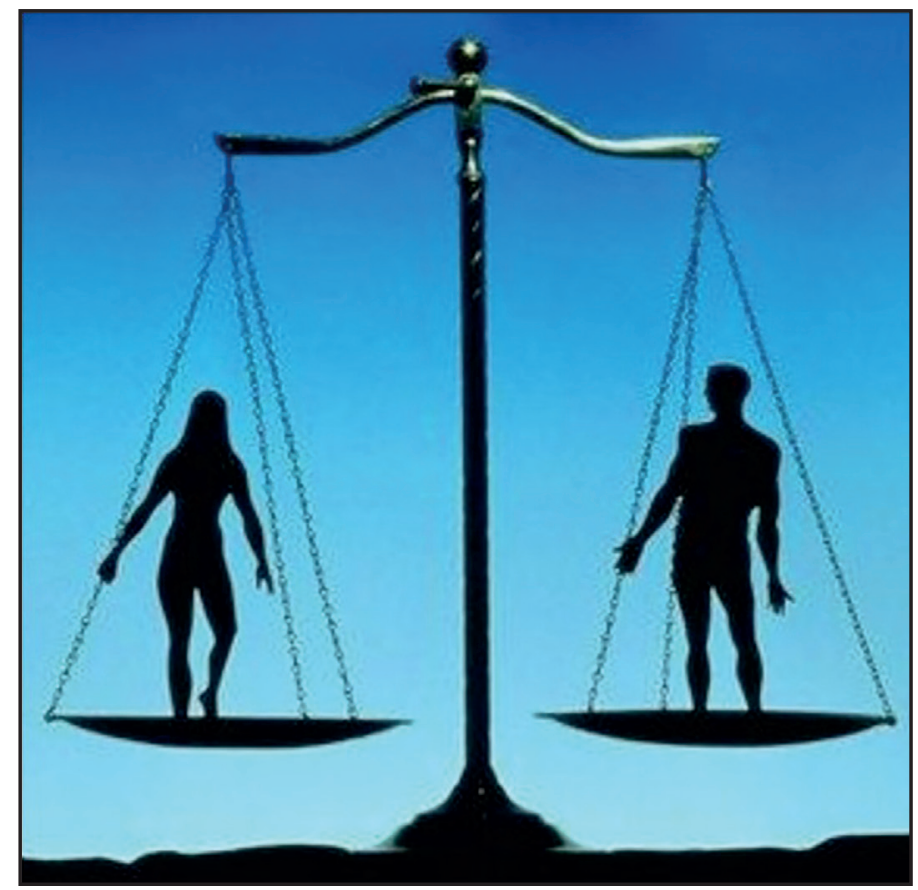

https://laecita.wordpress.com/2011/03/10/democracia-y-equidad-de-genero-otra-asignatura-pendiente/

\title{
DEMOCRACIA Y GÉNERO
}

\author{
Ullauri Donoso, Narcisa; Riera, Jéssica \\ Correspondencia: nullauri@uazuay.edu.ec; \\ jessybela17@gmail.com
}




\section{Resumen}

Este artículo pretende analizar la participación de la mujer en la construcción del Estado nación, el proceso de desarrollo que la mujer ha tenido que librar para conseguir el derecho al voto.

La coyuntura entre los hechos y las protagonistas ayudan a la participación femenina en democracia en el Ecuador, hecho que se va consolidando en la historia política ecuatoriana y en especial en estos 187 años de vida republicana.

Las actoras reconocidas y las mujeres desconocidas forman parte de la construcción de una democracia, que con el derecho al voto y la oportunidad de elegir y ser elegidas se consolida.

Palabras clave: voto, género, paridad, equidad, sufragio, constitución. 


\section{DEMOCRACY AND GENDER}

\section{Abstract}

The aim of this article is to analyze the participation of women in the construction of a nation-state and the development process that women have had to battle to obtain the right to vote.

The juncture between facts and protagonists has helped women's participation in democracy in Ecuador. This fact is being consolidated in the Ecuadorian political history, and especially in these 187 years of republican life.

Recognized protagonists and unknown women are part of building a democracy, which is consolidated through the right to vote and the opportunity to choose and be elected.

Keywords: ballot, gender, parity, equity, vote, constitution. 


\section{INTRODUCCIÓN}

El proceso de la participación de la mujer en la construcción de la democracia en el Ecuador republicano está marcado por acontecimientos y personas que impulsaron este sueño. Las constituciones políticas, a finales del siglo XIX e inicios del siglo XX, van abriendo un camino a este proceso democrático, partiendo desde las ideas de cambio con el movimiento de la llustración, la Revolución Liberal, el imaginario del progreso en el siglo $\mathrm{XX}$, se ven consolidadas en el desarrollo con participación igualitaria y equidad de género.

Además de las leyes se encuentran mujeres que buscaron conseguir la igualdad en todos los ámbitos, su lucha hoy es la bandera de la democracia en el Ecuador, Matilde Hidalgo, la primera mujer en votar en el Ecuador, Nela Martínez la primera diputada, y con ellas mujeres blancas, mestizas, indígenas que enriquecieron la historia del Ecuador con su participación política. Hoy se han convertido en iconos del proceso democrático para conseguir la equidad de género.

\section{LA COLONIA}

La época colonial marcó en la historia de la mujer ecuatoriana la vida de sumisión hacia el hombre; las mujeres indias, criollas, mestizas y chapetonas debían respeto y obediencia a la calidad de varón, en la persona del hermano mayor, padre, esposo y sacerdote. Los pocos referentes femeninos en la lucha política se han ido invisibilizando en la historia contada por hombres. 
La participación de la mujer en el siglo XIX viene dada con la idea de cambio, de un mundo diferente. Esta percepción de transformación es manejada por el movimiento de la llustración, este imaginario tiene una fuerte presencia en la primera mitad del siglo XIX, lo encontramos como referente, vigente en la Independencia. El término daba cabida a ideas y estilos nuevos, reformando las instituciones nacionales básicas o modificando las nuevas costumbres, bajo este paradigma la presencia de la mujer en la lucha por la independencia fue fundamental.

La mujer, en la consecución de este proceso libertario, ha sido poco estudiada. No se les ha dado el sitio que les corresponde a mujeres como Manuela Espejo, Josefa Tinajero y Checa, Mariana Matheu y Ascásubi, Manuela Cañizares y Álvarez, Rosa Zárate y Ontaneda, María Ontaneda y Larraín, María de la Vega y Nates, Rosa Montufar y Larrea y Manuela Quiroga y Coello, Antonia Salinas, Josefa Escarcha, María de la Cruz Vieyra, la "Costalona" o Estefa Campuzano, Rosa Solano, Margarita Orozco, Manuela Solís, entre otras; la historia poco las reconoce. Éstas próceres de la independencia vestían trajes de soldados lo que incluía enfrentar la muerte en campos de batalla, sin embargo su papel está impreso en sepia.

Además de estas mujeres ilustradas que participaron desde diversos espacios, se encuentran las traperas, que hacían la labor de remendar uniformes y curar heridas físicas y morales. Todas ellas dieron su vida por este sueño libertario y han pasado, a la historia escrita por hombres, a formar parte de la amnesia histórica, del olvido y el anonimato. 


\section{LA REPÚBLICA}

Una vez declarada la República del Ecuador, en el año de 1830, se realizó la primera constituyente en Riobamba por el presidente Juan José Flores, en la misma que el artículo 12 disponía: “...para ser considerado ciudadano tenía que tener 22 años, ser casado, saber leer y escribir, y tener bienes raíces por 300 pesos"1.

Con esta disposición las mujeres quedaron automáticamente al margen, no tenían educación, propiedades y estaban apegadas a los hombres de la familia; incluso las mujeres pudientes no optaron por la ciudadanía, como es el caso de Mercedes Jijón, esposa del Presidente Flores quién recibió clases privadas, lo cual le ayudaría para la posterior obtención de la ciudadanía, pero para realizarlo necesitaba el consentimiento de su esposo (Morales, 2009).

En la segunda constitución, la de 1835, realizada por Vicente Rocafuerte, la mujer seguía siendo destinada a labores domésticas y de sumisión, las de posibilidades económicas eran educadas únicamente en su hogar. Sin embargo, en este periodo aparece el Colegio Santa María del Socorro en el Beaterio en Quito como el primer plantel femenino público para niñas pobres, con su directora Josefa Carrión Ordóñez, primera maestra seglar en el país.

El referente de educación de la época es Dolores Veintimilla, escritora, que cuestionó el poder político y el papel de la iglesia, lo que le costó que la iglesia la considerara "sectaria" y le negara la sepultura eclesiástica.

1 Primera Constitución del Ecuador. 
En la séptima constitución, la convención de 1861, el Vicepresidente de la Convención, Mariano Cueva, sugiere tomar en cuenta a los menores de 21 años y las mujeres; convirtiéndose en la primera vez en que la mujer es mencionada para la posibilidad de ejercer el voto. Por segunda ocasión el Vicepresidente se pronuncia a favor de las mujeres: "-¿Por qué también no declaramos la ciudadanía respecto a las mujeres que tiene dotes apreciables en la imaginación y en el corazón?". Nadie se pronuncia y esta moción no avanza y no es tomada en cuenta (Morales, 2009).

En 1869, en la presidencia de García Moreno, con la octava Constitución conocida como "la Carta Negra" se imponía la religión católica, y la educación de las mujeres estaba a cargo de las monjas de los Sagrados Corazones y monjas de la Providencia.

En la Asamblea de 1878 se nombra como presidente interino a Ignacio de Veintimilla y junto a él aparece su sobrina Marietta, quien tiene un rol importante en el gobierno., es ella la cuestionadora de la democracia. Su pensamiento se encuentra en su ensayo: "Páginas del Ecuador" en el que deja notar que el problema se basa en los principios de: "democracia, liberalismo, tolerancia, coparticipación partidista y apoyo popular". Ésta mujer se convierte en la ideóloga femenina, tras la administración de Veintimilla. (Giabbai, 1998).

Esta pensadora muestra una perspectiva nueva de la mujer, basada en modelos históricos de Manuela Sáenz y Madame Ronald, que la lleva a obtener la fama de librepensadora, fue ella quien construyó el camino para la mujer en la política. 
Marietta conocida como "La Generala", estaba al frente a las estrategias de batalla, dirigiendo tropas al igual que en contra del clero y el partido conservador. Su coraje se manifiesta en el siguiente párrafo: "En el bolsillo de mi vestido llevaba un revólver cargado. Cuando me puse frente al Ejército, hice juramento de quitarme la vida en el posible instante que cayera en manos de esa gente ebria y soez. Esto da a mi espíritu completa serenidad". (Giabbai, 1998).

La Generala defiende a la mujer y la describe como: justicia, sacrificio, piedad, y caridad:

Nutrido el cerebro femenino de conocimientos útiles y nociones generales en armonía con el progreso, ¿será posible para el hombre... no encontrar algo de lo que le falta en el consejo de su hermana, madre o esposa?... Monstruos han inclinado la cabeza ante el mandato de una débil mujer, y júzguese la influencia que tendrán mañana en los humanos negocios las personas instruidas de nuestro sexo". (Giabbai, 1998).

En la Convención de 1883 - 1884, se designa Presidente de la República a Plácido Caamaño y se expidió la décima Constitución ecuatoriana. El tema de mayor interés y debate que se dio fue el voto de la mujer; los conservadores se opusieron a la igualdad de derechos y defendieron la idea de que el voto es solo para determinadas personas en donde no se incluye mujeres y niños. Por otro lado Remigio Crespo acepta el voto para la mujer, pero únicamente a aquellas que sepan leer y escribir. La Asamblea se mantuvo en debate debido al tema del sufragio universal y sus resultados no fueron favorables, pues se estipuló: "Para ser ciudadano se requiere saber 
leer y escribir, ser varón de veintiún años, ó ser ó haber sido casado" 2 .

Al inicio de la Revolución Liberal, a partir de 1895 se le encomendó la Jefatura Suprema al General Eloy Alfaro, las mujeres participaron en grandes batallas y fueron premiadas personalmente por el General como: Joaquina Galarza, ascendida a coronel, María Gamarra de Hidalgo y Filomena Chávez de Duque. Este fue el punto de partida con el que Alfaro comienza a dar trato a las mujeres e inicia firmando un decreto, el 7 de junio de 1895, en el cual permitía la matrícula de Aurelia Palmieri en la Escuela de Medicina de Guayaquil. En ese mismo año se estableció la escuela de Telegrafistas. (Morales, 2009).

En el Congreso de 1906, se permitió el voto de la mujer, quedando de la siguiente manera: "Para ser ciudadano se requiere la edad de veintiún años, saber leer y escribir"3.

Al estipular únicamente "saber leer y escribir", se podía tomar en cuenta a las mujeres que tenían estas características.

Es en estos momentos en que en el Ecuador aparecen mujeres respetadas por sus ideas de cambio y nuevos roles, una de ellas es Zoila Ugarte de Landívar, escritora, que a través de "La prensa" exponía su desacuerdo con Alfaro en el concepto de liberalismo. Desde este periódico abogó por las mujeres y su derecho al voto, al asumir la dirección de la revista "La Mujer", fue la primera en denominarse "feminista". (Morales, 2010).

2 Artículo 9. Décima Constitución ecuatoriana.

3 Artículo 13. Constitución Décima Segunda. 
En la presidencia de Leonidas Plaza el discurso cambia drásticamente, se habla sobre el feminismo y el Presidente demuestra su defensa hacia las mujeres, resaltando características y cualidades. Luego, con el congreso de 1906, la mujer abría su camino al derecho del voto.

De esta manera se va consolidando el derecho de la mujer al sufragio, que se ve cristalizado en el congreso de 1910, con una propuesta reformatoria a la Ley de Elecciones, que dispone en los artículos uno y tres el derecho al voto:

Art 1. "Son Electores todos los ecuatorianos (hombre o mujer) que tengan las cualidades prescritas en la Constitución y en las Leyes"4.

Art. 3 "Facúltase al Poder Ejecutivo para que expida los Reglamentos que creyere necesarios para que la mujer pueda ejercer el derecho electoral" ${ }^{5}$.

La propuesta fue debatida en toda la sesión, sin embargo no hubo cambio alguno.

Luego de varios acontecimientos y debates sobre el derecho al sufragio para la mujer, muchas mujeres empiezan a reclamar la igualdad de derechos entre hombres y mujeres.

La primera ecuatoriana que hizo prevalecer este derecho fue Matilde Hidalgo Navarro que fue la primera mujer en el país graduada de medicina en la Universidad de Cuenca. Matilde, basada en la Constitución de 1906, decidió apoyar el liberalismo. El 2 de mayo de 1924, 4 Reforma para el artículo 1 de la Décima Segunda Constitución-1906

5 Reforma para el artículo 13 de la Décima Segunda Constitución-1906 
Matilde se acercó a inscribirse en la Junta Electoral de Machala, donde le negaron esta posibilidad, sosteniendo que el voto era únicamente para varones. Sin embargo, mediante un alegato jurídico, ella quedó empadronada ese mismo día. (Vega, 1990).

En la sesión del 9 de Junio de 1924 se resolvió que las mujeres tenían derecho al voto y por consiguiente podían elegir y ser elegidas, cumpliendo con los requisitos que impone la Constitución.

Así esta mujer lojana de amplia trayectoria intelectual se convirtió en la primera mujer en sufragar en el Ecuador.

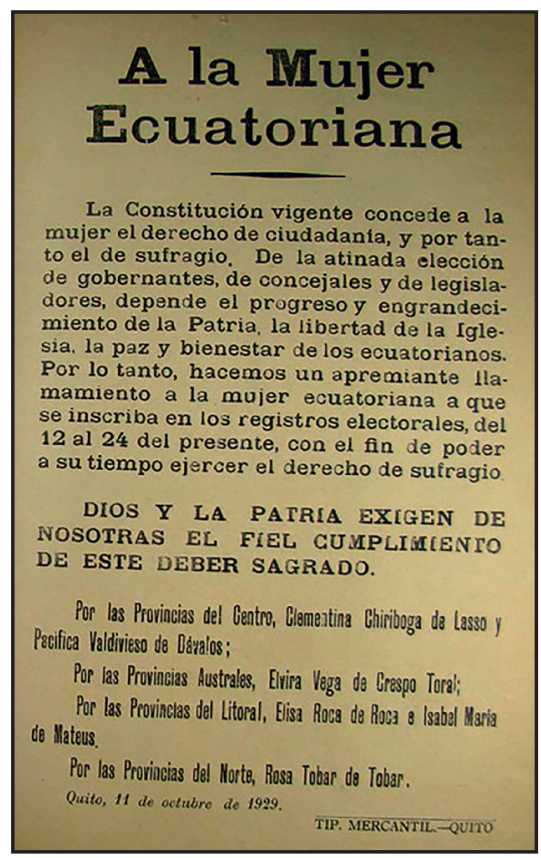

Fuente: Biblioteca Aurelio Espinoza Pólit 
Este derecho se consolida con la Constituyente de 1928 , al otorgar el derecho al voto a las mujeres mayores de 21 años que supieran leer y escribir.

No obstante se mantuvo la prohibición de que los analfabetos votaran. La mayoría de la población era analfabeta por lo que quedaba excluida de la participación electoral. Fue entonces cuando las mujeres con educación inician la alfabetización en sus haciendas y en las ciudades con grupos pequeños de 3 o 4 niñas y mujeres adultas.

Para las elecciones de 1929, ya hay células femeninas que buscan concientizar a la mujer en la importancia del voto. Los periódicos locales no publican los llamamientos a empadronarse, para ello se recurre a medios alternativos como las hojas volantes.

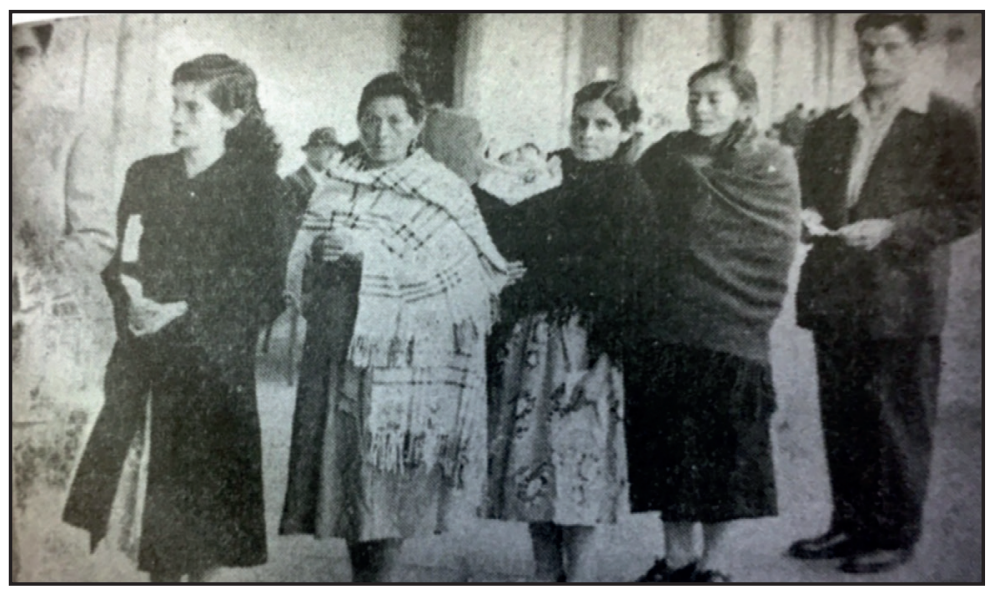

Fuente: Raquel Morales, Historia del voto femenino en el Ecuador 
Las mujeres empiezan a agruparse en comités femeninos electorales y a incursionar en la vida política. En 1928 se crea la Comisión Interamericana de la Mujer (CIM), que promovía la participación política de la mujer. Estos acontecimientos desencadenan que en 1929, el Ecuador sea el primer país latinoamericano en aprobar el voto de la mujer.

El partido socialista, integra en sus filas a mujeres, quienes hacen valer sus derechos y es este partido que en 1930, propone el nombre de Bertha Valverde Álvarez, como candidata a Concejal de Guayaquil. Así oficialmente la mujer incursiona en la vida política del país participando como candidata en un acto de elección popular.

En 1931 se registra por primera vez en las urnas un aproximado de 14000 mujeres que ejercían su derecho al voto. Las mujeres, en su mayoría, participaban en campañas políticas.

En 1945, en la asamblea Constituyente, en el artículo 15 se dispone que todo ecuatoriano, hombre o mujer de 18 años, que sepa leer y escribir, es ciudadano.

Esta condición "saber leer y escribir" fue muy utilizada en las Constituciones anteriores, lo cual no significó un cambio mayor debido a la carencia de educación de las mujeres.

En esta década las mujeres consiguen logros importantes, el movimiento indígena se ve representado por Dolores Cacuango y Tránsito Amaguaña, quienes lideran los Congresos de Indios, conformando alianzas con el partido Comunista, buscando reivindicaciones para los pueblos indígenas. (Grijalva, 2006). 
Por otro lado, en 1945 los trabajadores del Ecuador designaron como diputado funcional a la asamblea constituyente a Pedro Saad, con su primer suplente, Segundo Ramos y nombraron como segundo suplente a Nela Martínez. Este suceso convirtió a Nela en la primera mujer Diputada en el país, además la primera en ser miembro del comité ejecutivo de un partido Comunista del Ecuador. (Pimentel, 2008).

En 1960 se creó la Unión Nacional de Mujeres Ecuatorianas (UNME), que tenía como objetivo mejorar las condiciones de vida de la mujer y erradicar la discriminación en la parte laboral y legal.

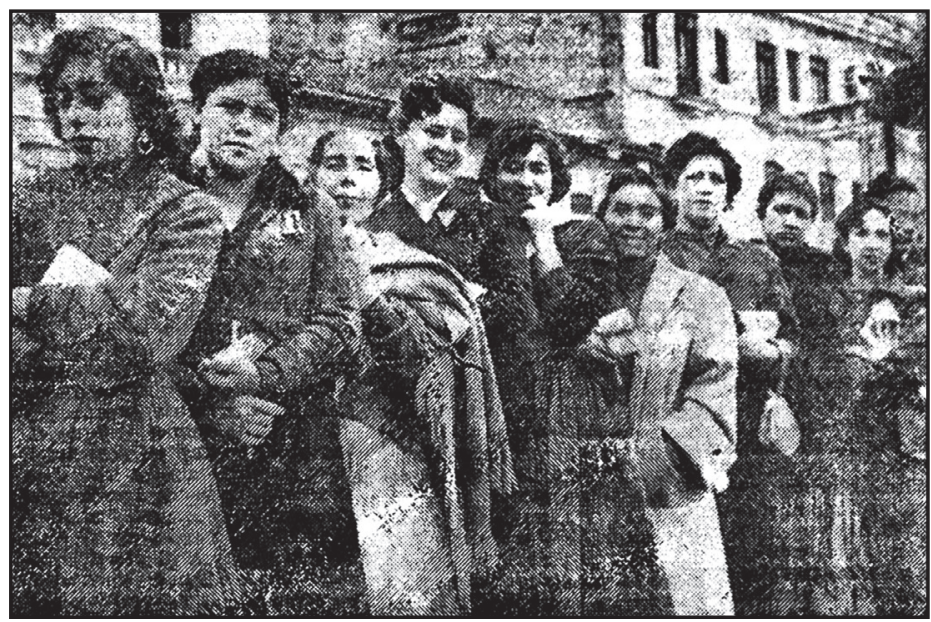

Fuente: Raquel Morales, Historia del voto femenino en el Ecuador

Isabel Robalino Bolle, legisladora de la asamblea y senadora, propone el voto obligatorio para la mujer, con el objetivo de disminuir la discriminación en la parte política; pues hasta ese momento, la mujer tenía voto facultativo. 
En la Constitución de 1967 se establece que el voto es un deber cívico obligatorio para hombres y mujeres; lo que arrojó un incremento del $50 \%$ de mujeres empadronadas para las elecciones en comparación con las inmediatamente anteriores.

En la Constitución de 1979 se dispone lo siguiente a favor de la mujer:

"Art. 19, No. 4: La igualdad ante la ley.

Se prohíbe toda discriminación por motivos de raza, color, sexo...

La mujer cualquiera sea su estado civil, tiene iguales derechos y oportunidades que el hombre en todos los órdenes de la vida pública, privada, y familiar, especialmente en lo civil, político, social y cultural".

Esta Constitución establece la obligatoriedad del voto; la edad para ejercer el sufragio se fija en 18 años y se incluye a la población analfabeta.

En la década de 1990 dos mujeres ocupan un rol fundamental en el congreso: Susana González fue la primera mujer Presidenta del Congreso y Nina Pacari la primera Vicepresidenta del Congreso. En la misma década, en 1996, se eligió a la primera mujer Vicepresidenta: Rosalía Arteaga, quien se convirtió en la primera mujer presidente del Ecuador en 1997. En el 2006 una mujer tercia por primera vez en las elecciones a la primera magistratura, con Cyntia Viteri. Así la batalla iniciada por Matilde Hidalgo ha durado un siglo y ha concluido parcialmente, pero quedan otros espacios donde se tiene que seguir luchando para lograr la equidad de género. 


\section{Conclusiones}

La participación política de la mujer en los distintos espacios se va consolidando en el siglo XX. Al romperse la manipulación clerical en la Revolución Liberal, con las primeras mujeres profesionales, con la participación de la mujer en los procesos educativos formales e informales, la mujer va construyendo una posición que no solamente se refleja en política sino también en la academia, en la producción. La mujer ocupa posiciones trascendentales, que antes estuvieron destinadas exclusivamente al hombre. La lucha por el derecho al voto abrió la puerta para construir nuevos espacios para las mujeres, estos espacios se fueron logrando desde el $20 \%$, luego $30 \%$ aumentando paulatinamente, para incorporar hasta el $50 \%$ de la participación, cumpliendo con los principios de paridad y alternancia.

Este artículo recorre el camino trazado por las mujeres ecuatorianas, iconos del feminismo, muchas de ellas no constan, dejando abierta la posibilidad a un estudio más profundo sobre la participación política de la mujer, tema del que muy poco se ha escrito. 


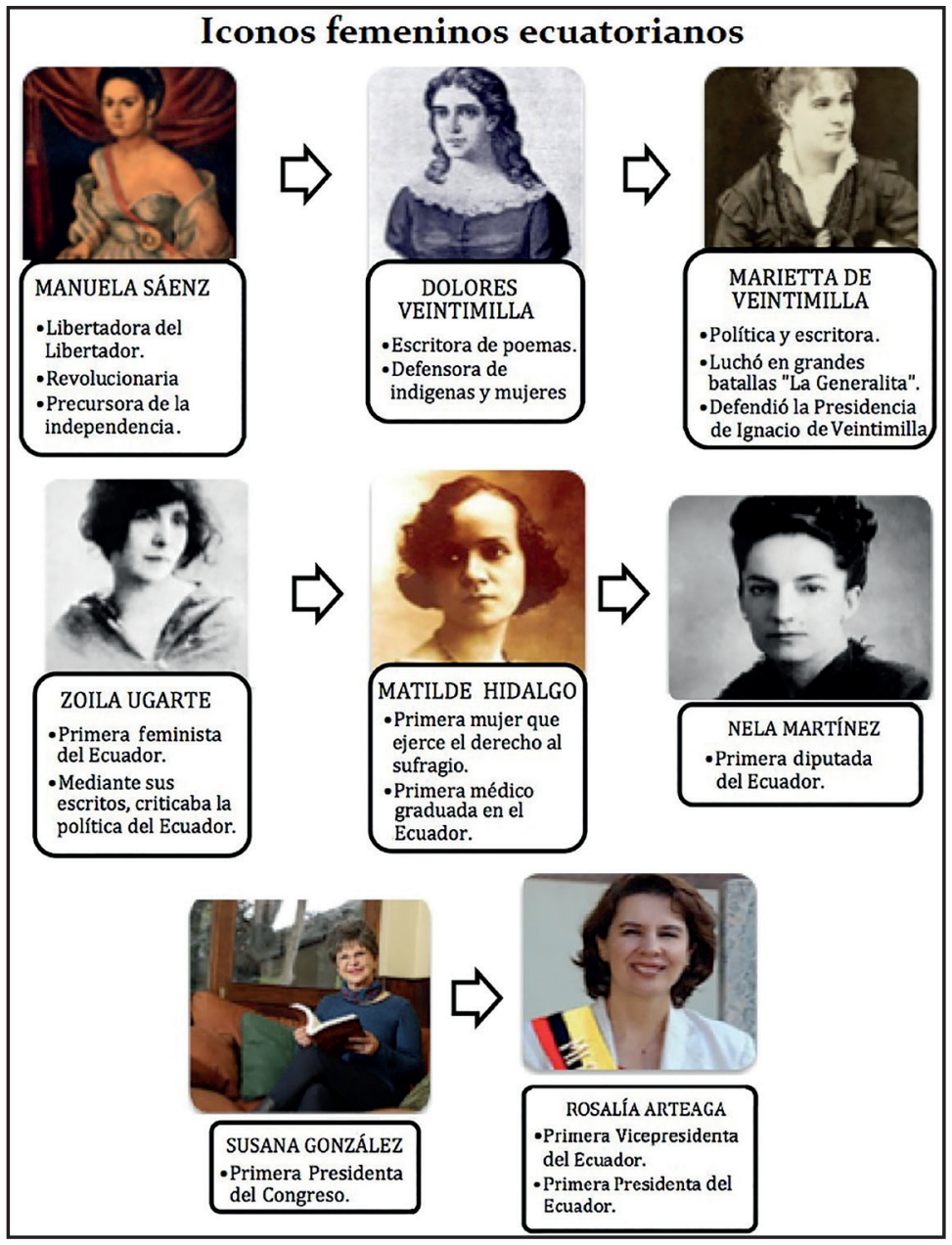




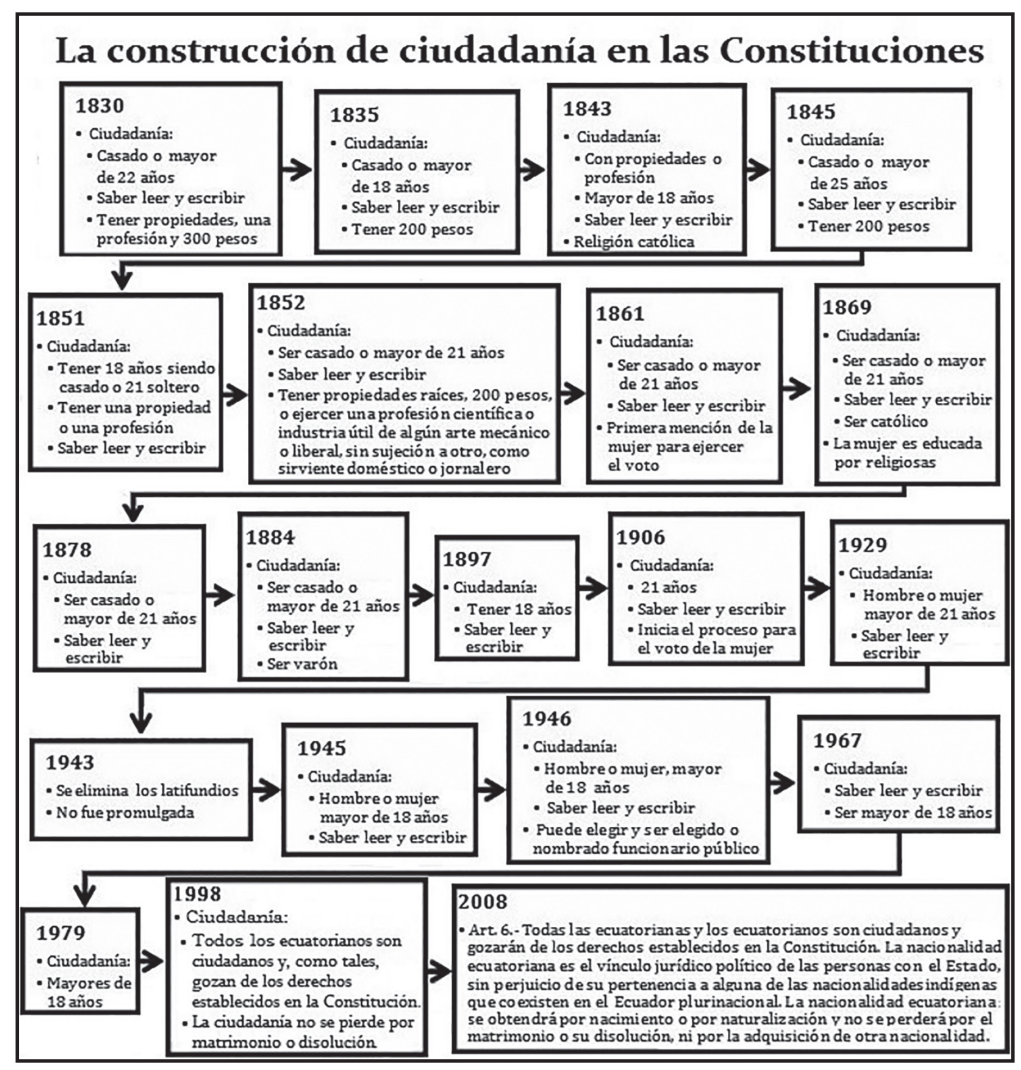




\section{Bibliografía}

EL COMERCIO. (8 de marzo de 2016). 10 MUJERES IMPRESCINDIBLES EN LA CULTURA ECUATORIANA. ECUADOR.

Giabbai, G. d. (1998). Marietta. Quito, Pichincha, Ecuador: Banco Central del Ecuador.

Grijalva, C. M. (2006). Tránsito Amaguaña. Quito, Pichincha, Ecuador: Banco Central del Ecuador.

Morales, R. R. (2005). Dolores Cacuango. (G. Landázuri, Ed.) Quito, Pichincha, Ecuador: Banco Central del Ecuador.

Morales, R. R. (2009). Historia del voto femenino en el Ecuador (1ra Edición ed.). (R. R. M., Ed.) Quito, Pichincha, Ecuador: Consejo Nacional de las Mujeres (CONAMU).

Morales, R. R. (2010). Zoila Ugarte de Landívar. Quito, Pichincha, Ecuador: BANCO CENTRALDELECUADOR.

Pimentel, R. P. (08 de 05 de 2008). Diccionario Biográfico del Ecuador. Recuperado el Febrero de 2017, de Sitio Web de Diccionario Biográfico del Ecuador: http:// www.diccionariobiograficoecuador.com/tomos/tomo16/ m4.htm

Vega, M. J. (1990). EL VOTO FEMENINO. En T. S. Electoral, Análisis de Procesos Electorales. Quito: Corporación Editora Nacional. 